[4] И. М. Гельфанд, Обобщенные случайные проиессы, Докл. Акад. Наук CCGP 100,5 (1955), p. $852-856$.

[5] P. R. Halmos, Measure theory, New York 1950

[6] K. Ito, Stationary random distributions, Mem. of the Coll. of Sci. Kyoto Univ., Ser. A, 28.3 (1954), p. $209-223$.

[7] А. М. Яглом, Коррелячионная теория прочессов со случайныци стачионарными п-ми прирощениями, Мат. сборник 37 (1) (1955), p. 141-196.

[8] S. Łojasiewicz, J. Wloka and Z. Zieleźny, Über eine Definition des Wertes einer Distribution, Bull. Acad. Pol. Sci., Cl. III.3, No 9 (1955), p. $479-480$.

[9] J. Mikusiński, Sur la méthode de Laurent-Schwartz et sur la convergence faible, Fund. Math. 35 (1948), p. $235 \cdot 241$.

[10] - Uñe définition de distribution, Bull Acad. Pol. Sci., Cl. III.3, No 11 (1955), p. $589-591$.

[11] J. Mikusiński and R. Sikorski, Distributions theory (to be published).

[12] К. У рбаник, Случайные прочессы, реализачии которых лвлялотся обобщенными функииями, Теория вероятностей I (1956), p. 146-149.

INSTYTUT MATEMATYCZNY POLSKIEJ AKADEMII NAUK

MATH

Regu par la Rédaction le 12. 9. 1956

\section{Some remarks on the convergence of functionals on bases}

by

W. ORLICZ and Z. CIESIELSKI (Poznań)

1. In this paper $X$ denotes a Banach space, unless explicitly stated otherwise.

We shall say that a subset $B$ of the set $A \subset X$ is a linear rational basis (briefly - LRB) of the set $A$ if $x \in A$ implies

$$
x=\sum_{i=1}^{m} \alpha_{i} x_{i},
$$

where $x_{i} \in B, \alpha_{i}$ are rational numbers and $m$ is a positive integer depending on $x$.

If the representation (*) is unique for each $x \in A$ then the set $B$ is called a rational Hamel basis of the set $A$.

We shall say that the subset $B^{*}$ of the set $A \subset X$ is a convex rational basis (briefly - CRB) of the set $A$ if there exists a point $a \in B^{*}$ and a real number $M>0$ such that $x \in A$ implies

$$
\dot{x}=\sum_{i=1}^{m} \beta_{i}\left(x_{i}-a\right)+a,
$$

where $x_{i} \in B^{*}, \beta_{i} \geqslant 0$ are rational numbers satisfying the condition $\beta_{1}+\ldots+\beta_{m} \leqslant M$ with a positive integer $m$, depending on $x$.

We observe that every CRB of the set $A$ is an LRB of this set.

We say that the functional $\xi$ defined in a convex set $D \subset X$ is a convex functional in $D$ if for any $x, y \in D$ we have the inequality

$$
\xi(\lambda x+\mu y) \leqslant \lambda \xi(x)+\mu \xi(y),
$$

where $\lambda \geqslant 0$ and $\mu \geqslant 0$ are arbitrary rational numbers satisfying the condition $\lambda+\mu=1$.

If the functional $\xi$ is continuous in $D$ the inequality $\left(\begin{array}{c}* * \\ *\end{array}\right)$ is satisfied for real $\lambda, \mu$.

1. 1. We denote by $\bar{K}\left(x_{0}, r\right)$ the closure of the sphere $K\left(x_{0}, r\right)$. Further we use the following terminology.

An arbitrary functional $\xi$ is uniformly bounded in a set $A$ if there exists a constant $G$ such that $x \in A$ implies $|\xi(x)|<G$, where $G$ does not depend on $x$. 
$\xi$ is locally uniformly bounded in a region $D^{1}$ ) if there exists for every $x \in D$ a neighbourhood $K$ such that $\xi$ is uniformly bounded in $K$.

The sequence $\left\{\xi_{n}\right\}$ is bounded at the point $x$ if there exists a constant $G(x)$ such that $\left|\xi_{n}(x)\right|<G(x)$ for $n=1,2, \ldots$

$\left\{\xi_{n}\right\}$ is bounded in a set $A$ if it is bounded at each point of this set.

$\left\{\xi_{n}\right\}$ is uniformly bounded in a set $A$ if there exists a constant $G$ such that $x \in A$ implies $\left|\xi_{n}(x)\right|<G$ for $n=1,2, \ldots$

$\left\{\xi_{n}\right\}$ is locally uniformly bounded in a region $D$ if there exists for every $x \in D$ a neighbourhood $K$ such that $\left\{\xi_{n}\right\}$ is uniformly bounded in $K$.

We shall also use the notion of the boundedness above (below) of a functional $\xi$ and a sequence $\left\{\xi_{n}\right\}$. The meaning of this terminology will be analogical to that given above.

1.2. Let the functional $\xi$ defined and convex in a convex region $D$ be unitormly bounded above in a sphere $K\left(x_{0}, r\right) \subset D$ by a constant $G$. Then it is uniformly bounded below in this sphere by a constant $2 \xi\left(x_{0}\right)-G$.

Since $y \in K\left(x_{0}, r\right)$ implies $\xi(y)<G$, we have for $x \in K\left(x_{0}, r\right)$

$$
\xi(x) \geqslant 2 \xi\left(x_{0}\right)-\xi\left(2 x_{0}-x\right)>2 \xi\left(x_{0}\right)-G .
$$

1.3. Let the functional $\xi$ defined and convex in a convex region $D$ be uniformly bounded above in a sphere $K\left(x_{0}, r\right) \subset D$. Then it is continuous at the point $x_{0}$.

Suppose $\xi(x)<G$ for $x \in K\left(x_{0}, r\right)$. Then one may easily prove that we have the inequalities

$$
\frac{G-\xi\left(x_{0}\right)}{n}>\xi\left(x_{0}+h\right)-\xi\left(x_{0}\right) \geqslant \xi\left(x_{0}\right)-\xi\left(x_{0}-h\right)>\frac{\xi\left(x_{0}\right)-G}{n},
$$

where $n$ and $h \in X$ are such that $x_{0} \pm n h \in K\left(x_{0}, r\right)$ (see [4], p. 92-93). Thus for every $\varepsilon>0$ there exists a $\delta>0$ such that $\|h\|<\delta$ implies $\left|\xi\left(x_{0}\right)-\xi\left(x_{0}+h\right)\right|<\varepsilon$.

1.4. If the functional $\xi$ is continuous, convex and uniformly bounded above in $\bar{K}\left(x_{0}, r\right)$, then it satisfies for every $0<\gamma<1$ the Lipschitz condition in the sphere $K\left(x_{0}, \gamma r\right)$.

Suppose $\left\|y_{1}-x_{0}\right\|=r$ and $\left\|y_{2}-x_{0}\right\|=\gamma r$. Our assumptions imply the inequalities $2 \xi\left(x_{0}\right)-G<\xi\left(y_{1}\right)<G$ and $-G<-\xi\left(y_{2}\right)<G-2 \xi\left(x_{0}\right)$. Hence $2\left(\xi\left(x_{0}\right)-G\right)<\xi\left(y_{1}\right)-\xi\left(y_{2}\right)<2\left(G-\xi\left(x_{0}\right)\right)$.

Since $r(1-\gamma) \leqslant\left\|y_{1}-y_{2}\right\| \leqslant r(1+\gamma)$, we have

$$
\frac{2\left(\xi\left(x_{0}\right)-G\right)}{r(1+\gamma)}<\frac{\xi\left(y_{1}\right)-\xi\left(y_{2}\right)}{\left\|y_{1}-y_{2}\right\|}<\frac{2\left(G-\xi\left(x_{0}\right)\right)}{r(1-\gamma)} .
$$

Let $x_{1}, x_{2}$ be arbitrary two points of the sphere $K\left(x_{0}, \gamma r\right)$; we consider four different points $y_{i}=x_{1}+t_{i}\left(x_{2}-x_{1}\right)$ such that $\left\|y_{i}-x_{0}\right\|=r$ for

1) E. g. an open set. $i=1,4,\left\|y_{i}-x_{0}\right\|=\gamma r$ for $i=2,3$. The values of the parameters $t_{1}, t_{2}, t_{3}$ and $t_{4}$, corresponding to the points $y_{1}, y_{2}, y_{3}$ and $y_{4}$ respectively, satisfy the conditions $t_{1} t_{4}<0$ and $t_{2} t_{3}<0$. We take $t_{1}<0$ and $t_{2}<0$. Since $y_{3}, y_{4} \notin K\left(x_{0}, \gamma r\right)$ and $t_{3}>0, t_{4}>0$, we have $t_{3}>1, t_{4}>1$. We observe that $t_{1}<t_{2}<0<1<t_{3}<t_{4}$. Since the real function $\varphi(t)=\xi\left(x_{1}+t\left(x_{2}-x_{1}\right)\right)$ is convex in the closed interval $\left\langle t_{1}, t_{4}\right\rangle$, we have the inequalities (compare [4], p. 93-94)

$$
\frac{\varphi\left(t_{2}\right)-\varphi\left(t_{1}\right)}{t_{2}-t_{1}} \leqslant \varphi(1)-\varphi(0) \leqslant \frac{\varphi\left(t_{4}\right)-\varphi\left(t_{3}\right)}{t_{4}-t_{3}} .
$$

Hence follow the inequalities

$$
\frac{\xi\left(y_{2}\right)-\xi\left(y_{1}\right)}{\left\|y_{2}-y_{1}\right\|} \leqslant \frac{\xi\left(x_{2}\right)-\xi\left(x_{1}\right)}{\left\|x_{2}-x_{1}\right\|} \leqslant \frac{\xi\left(y_{4}\right)-\xi\left(y_{3}\right)}{\left\|y_{4}-y_{3}\right\|} .
$$

The above inequalities imply

$$
\left|\xi\left(x_{2}\right)-\xi\left(x_{1}\right)\right|<\frac{2\left(G-\xi\left(x_{0}\right)\right)}{r(1-\gamma)}\left\|x_{2}-x_{1}\right\|
$$

for arbitrary $x_{1}, \dot{x_{2}} \in K\left(x_{0}, \gamma r\right)$.

2. If the functional $\xi$ defined and convex in a convex region $D \subset X$ is uniformly bounded above in a CRB of D then it satisfies the local Lipschitz condition and consequently is locally uniformly continuous in $D$.

Let $r>0$ be such that $K(a, r) \subset D$. Then there exists a number $\varrho>0$ such that every $y \in K(a, \varrho)$ is of the form $y=s(x-a)+a$, where $x \in K(a, r)$ and $s=1 / M$. Moreover, the constant $s$ may be supposed to be rational. Since $z \in B^{*}$ implies $\xi(z)<G$, we have

$$
\begin{aligned}
\xi(y) & =\xi(s(x-a)+a)=\xi\left(\sum_{i=1}^{m} s \beta_{i}\left(x_{i}-a\right)+a\right) \\
& \leqslant \sum_{i=1}^{m} s \beta_{i} \xi\left(x_{i}\right)+\left(1-\sum_{i=1}^{m} s \beta_{i}\right) \xi(a)<G .
\end{aligned}
$$

Hence it follows that $y \in K(a, \varrho)$ implies $\xi(y)<G$. Let $x_{0}$ belong to $D-K(a, \varrho)$. Then there exist numbers $a>0, \beta>0$ and a point $b \epsilon D$ such that $\alpha+\beta=1$ and $x_{0}=\alpha a+\beta b$. The point $x_{0}$ is an interior point of the cone

$$
S=\{x: x=p y+q b, y \in K(a, \varrho), p>0, q>0, p+q=1\} .
$$

In fact, there exists a $\delta>0$ such that $\left\|x-x_{0}\right\|<\delta$ implies $\|y-a\|<\varrho$. Thus we have, for $x \in K\left(x_{0}, \delta\right), \xi(x)<\max (G, \xi(b))$. Hence 1.2, 1.3 and 1.4 imply 2.

Studia Mathematica XVI 
2.1. The assumption of the completeness of the space $X$ is superfluous in 2 .

2.2. Let $\eta$ denote an additive functional defined in $X$ as follows. If $x$ belongs to a rational Hamel basis $B$ of the sphere $K(0,1)$ then $\eta(x)$ equals an arbitrary negative number. For $x \in K(0,1)-B$ we put $\eta(x)=$ $=\alpha_{1} \eta\left(x_{1}\right)+\alpha_{2} \eta\left(x_{2}\right)+\ldots+\alpha_{m} \eta\left(x_{m}\right)$, where $\alpha_{i}$ and $x_{i} \in B$ are given by $(*)$. Since the functional $\eta$ is additive, it is convex. Moreover, it is uniformly bounded above in an LRB of $K(0,1)$, but it is not continuous. It follows from 2 that the rational Hamel basis of the sphere $K(0,1)$ cannot be a CRB of the sphere $K(0,1)$.

3. Let the functional $\xi_{n}$ defined in a convex region $D$ be continuous and convex in $D$ for $n=1,2, \ldots$. Then if the sequence $\left\{\xi_{n}\right\}$ is bounded above in $a C R B$ of $D$ and

(a) if the sequence $\left\{\xi_{n}\right\}$ is bounded in a set dense in $D$ then it is locally uniformly bounded in $D$, and $\xi_{n}$ satisfies the local Lipschitz condition uniformly in $n$ (consequently $\xi_{n}$ are locally equicontinuous),

(b) if the sequence $\left\{\xi_{n}\right\}$ is convergent in a set dense in $D$ then it is convergent in the whole region $D$ to a convex and continuous functional $\xi$.

Ad (a). Our assumption implies $\xi_{n}(x)<G$ for $n=1,2, \ldots, x \in B^{*}$. Retaining the notation from the section 2 we may conclude that for every $y \in K(a, \varrho)$ there exists an $x \in D$ such that $y=s(x-a)+a$. Hence

$$
\begin{aligned}
\xi_{n}(y) & =\xi_{n}(s(x-a)+a)=\xi_{n}\left(\sum_{i=1}^{m} s \beta_{i}\left(x_{i}-a\right)+a\right) \\
& \leqslant \sum_{i=1}^{m} s \beta_{i} \xi_{n}\left(x_{i}\right)+\left(1-\sum_{i=1}^{m} s \beta_{i}\right) \xi_{n}(a) \\
& <\sum_{i=1}^{m} s \beta_{i} G\left(x_{i}\right)+\left(1-\sum_{i=1}^{m} s \beta_{i}\right) G(a) \\
& \leqslant \max \left(G\left(x_{1}\right), \ldots, G\left(x_{m}\right), G(a)\right)=G(y) .
\end{aligned}
$$

We consider the sphere $\bar{K}\left(a, \varrho^{*}\right) \subset K(a, \varrho)$. We write $F_{r}=$ $=\left\{x: \xi_{n}(x) \leqslant r, x \in K\left(a, \varrho^{*}\right), n=1,2, \ldots\right\}$ for $r=1,2, \ldots$ The sets $F_{r}$ are closed and $\bar{K}\left(a, \varrho^{*}\right)=F_{1} \subset F_{2} \subset \ldots$ Thus there exists an $r_{0}$ such that $F_{r_{0}} \supset K\left(y_{0}, \varrho^{\prime}\right)$. Hence we have $\xi_{n}(x)<r_{0}+1=G_{1}$ for $x \in K\left(y_{0}, \varrho^{\prime}\right)$ and $n=1,2, \ldots$ If $x_{0} \in K\left(y_{0}, \varrho^{\prime}\right)$, then the sequence $\left\{\xi_{n}\right\}$ is uniformly bounded above in a neighbourhood of $x_{0}$ and the theorem is proved. Now let us suppose $x_{0} \in D-K\left(y_{0}, \varrho^{\prime}\right)$. Let $H$ denote a set dense in $D$ such that $\left\{\xi_{n}\right\}$ is bounded in $H$. Then there exist a sphere $K\left(y_{1}, \varrho^{\prime \prime}\right) \subset K\left(y_{0}, \varrho^{\prime}\right)$, a point $z_{0} \in H$ and numbers $\alpha>0, \beta>0$ such that $\alpha+\beta=1$ and $x_{0}=\alpha y_{1}+\beta z_{0}$.
Now we repeat the arguments of the last part of the proof in the section 2. We obtain $\xi_{n}(x)<\max \left(G_{1}, \xi_{n}\left(z_{0}\right)\right)$ for $n=1,2, \ldots$ and $x \epsilon K\left(x_{0}, \delta\right)$. Since $\xi_{n}\left(z_{0}\right)<G\left(z_{0}\right)$, we have, for every $n$ and $x \in K\left(x_{0}, \delta\right), \xi_{n}(x)<$ $<\max \left(G_{1}, G\left(z_{0}\right)\right)=G\left(x_{0}\right)$.

Further let us observe that the sequence $\left\{\xi_{n}\right\}$ is locally uniformly bounded below in $D$. Indeed, there exists a point $z_{1} \in H$ such that $\left\|z_{1}-x_{0}\right\|<$ $<\delta / 4$ and the sequence $\left\{\xi_{n}\right\}$ is uniformly bounded below in the. sphere $K\left(z_{1}, \delta / 2\right)$. Since $x_{0} \in K\left(z_{1}, \delta / 2\right)$, there exists a sphere $\bar{K}\left(x_{0}, \delta_{1}\right) \subset K\left(z_{1}, \delta / 2\right)$. Thus there exists for $x \in \bar{K}\left(x_{0}, \delta_{1}\right)$ a constant $G^{*}\left(x_{0}\right)$ such that $\left|\xi_{n}(x)\right|<$ $<G^{*}\left(x_{0}\right)$ for $n=1,2, \ldots$ From 1.4 it follows that

$$
\frac{\left|\xi_{n}\left(x_{1}\right)-\xi_{n}\left(x_{2}\right)\right|}{\left\|x_{1}-x_{2}\right\|}<\frac{2\left(G^{*}\left(x_{0}\right)-\xi_{n}\left(x_{0}\right)\right)}{r(1-\gamma)}
$$

for $n=1,2, \ldots$ and for arbitrary $x_{1}, x_{2} \in K\left(x_{0}, \gamma \delta_{1}\right)$, where $0<\gamma<1$. Consequently,

$$
\left|\xi_{n}\left(x_{1}\right)-\xi_{n}\left(x_{2}\right)\right|<\frac{4 G^{*}\left(x_{0}\right)}{r(1-\gamma)}|| x_{1}-x_{2} \|
$$

in the sphere $K\left(x_{0}, \gamma \delta_{1}\right)$.

Ad (b). The proof is immediately obtained from (a).

3.1. If we suppose $X$ to be an arbitrary $(F)$-space, then lemmas 1.2 and 1.3 remain true. However, Theorem 2 must be formulated for spaces of type $(F)$ in a less general form.

If the functional $\xi$, defined and convex in a convex region $D$, is uniformly bounded above in a CRB of $D$ then it is locally uniformly continuous in $D$.

The method of the proof of the section 2 shows that $\xi$ is locally uniformly bounded in $D$. Let $x_{0}$ denote an arbitrary point of $D$. Applying 1.3 (with the same notation) we see that for every point $x_{1} \in K\left(x_{0}, r_{1}\right)$ (where $0<r_{1}<r$ ) the inequalities

$$
\frac{G-\xi\left(x_{1}\right)}{n}>\xi\left(x_{1}+h\right)-\xi\left(x_{1}\right) \geqslant \xi\left(x_{1}\right)-\xi\left(x_{1}-h\right)>\frac{\xi\left(x_{1}\right)-G}{n}
$$

hold for $h \epsilon X$ such that $\|n h\|<r-r_{1}$. From 1.2 and from the last inequalities follows the inequality

$$
\left|\xi\left(x_{1}\right)-\xi\left(x_{2}\right)\right|<\frac{2\left(G-\xi\left(x_{0}\right)\right)}{n},
$$

where $x_{2}=x_{1}+h$. Thus for every $\varepsilon>0$ there exists $\delta>0$ such that $\left\|x_{1}-x_{2}\right\|<\delta$ implies $\left|\xi\left(x_{1}\right)-\xi\left(x_{2}\right)\right|<\varepsilon$ for arbitrary $x_{1}, x_{2} \epsilon K\left(x_{0}, r_{1}\right)$.

3.2. The formulation of Theorem 3 for a space $X$ of type $(F)$ is similar to its formulation for a Banach space. In the case of $\left(F^{T}\right)$-spaces one 
must replace the local Lipschitz condition by the local equicontinuity in $D$ of the functionals $\xi_{n}$, as follows from the method of the proof of Theorem 3 and from 1.2 and 1.3 .

3.21. Let $\xi_{n}$ be a functional continuous and convex in $D$ for $n=1,2, \ldots$ and let the sequence $\left\{\xi_{n}\right\}$ be convergent (bounded) in a set dense in $D$. Then the sequence $\left\{\xi_{n}\right\}$ is either convergent (locally unitormly bounded) in whole $D$ or convergent (bounded above in) a set which is not $C R B$ of $D$.

3.22. If $D=X$ and if $U_{n}$ is a linear operation from $X$ to $Y, X, Y$ being Banach spaces, then the functional $\xi_{n}(x)=\left\|U_{n}(x)\right\|$ is convex and continuous on $X$ for $n=1,2, \ldots$ Then it is easily seen that if the sequence $\left\{\xi_{n}\right\}$ is bounded in an LRB of $X$ then it is also bounded in $X$. Moreover, the functionals $\xi_{\imath}$ are positive-homogeneous. Thus, since the sequence $\left\{\xi_{n}\right\}$ is locally uniformly bounded in a neighbourhood of zero (see section 3), it is also uniformly bounded in the unit sphere. Consequently, the well known Banach-Steinhaus theorem (see [3], p. 80) may be formulated more generally as follows:

A sequence of linear operations $\left\{U_{n}\right\}$ is either uniformly bounded in the unit sphere or bounded in a set which is not an LRB of $-X$.

3.23. Let $\xi=\xi\left(x_{1}, \ldots, x_{k}\right)$ denote a functional defined for $x_{i} \epsilon K\left(x_{i}^{0}, r_{i}\right)$ $C X_{i}, X_{i}$ being Banach spaces. If $\xi$ is a continuous and convex functional of the variable $x_{i}$ in $K\left(x_{i}^{0}, r_{i}\right)$ for $i=1,2, \ldots, k$, then it is a continuous functional of the point $\left(x_{1}, \ldots, x_{k}\right)$.

Write $K=K\left(x_{1}^{0}, r_{1}\right) \times \ldots \times K\left(x_{k}^{0}, r_{k}\right)$. We choose for every point $\left(x_{1}^{*}, \ldots, x_{k}^{*}\right) \in K$ an arbitrary sequence of points $\left(x_{1}^{n}, \ldots, x_{k}^{n}\right) \in K$ such that $x_{i}^{n} \rightarrow x_{i}^{*}$ for $i=1,2, \ldots, k$. Put $\xi_{n}\left(x_{1}\right)=\xi\left(x_{1}, x_{2}^{n}\right)$. Then for every $x_{1} \in K\left(x_{1}^{0}, r_{1}\right), \xi_{n}\left(x_{1}\right) \rightarrow \xi\left(x_{1}, x_{2}^{*}\right)$. According to section $3, \xi_{n}$ are equicontinuous at the point $x_{1}^{*}$. Hence $\xi\left(x_{1}^{n}, x_{2}^{n}\right) \rightarrow \xi\left(x_{1}^{*}, x_{2}^{*}\right)$. This implies, by an easy induction $\xi\left(x_{1}^{n}, \ldots, x_{k}^{n}\right) \rightarrow \xi\left(x_{1}^{*}, \ldots, x_{k}^{*}\right)$.

3.24. Let the functional $\xi_{n}$ satisfy for $n=1,2, \ldots$ the same assumptions as the functional $\xi$ in 3.23 .

If $\xi_{n}\left(x_{1}, \ldots, x_{k}\right) \rightarrow \xi_{0}\left(x_{1}, \ldots, x_{k}\right)$ in $K$, then the $\xi_{n}$ are equicontinuqus at an arbitrary point $\left(x_{1}, \ldots, x_{k}\right) \epsilon K$.

Let $\left(x_{1}^{*}, \ldots, x_{k}^{*}\right)$ denote a fixed point belonging to $K$, and $\left(x_{1}^{n}, \ldots, x_{k}^{n}\right) \in K$ for $n=1,2, \ldots$ an arbitrary point such that $x_{i}^{n} \rightarrow x_{i}^{*}$ for $i=1,2, \ldots, k$. We put $\eta_{n}\left(x_{1}, \ldots, x_{i}\right)=\xi_{n}\left(x_{1}, \ldots, x_{i}, x_{i+1}^{*}, \ldots, x_{k}^{*}\right)$. According to section $3, \eta_{n}\left(x_{1}\right)$ are equicontinuous functionals of the variable $x_{1}$ at the point $x_{1}^{*}$. Thus $\eta_{n}\left(x_{1}^{n}\right) \rightarrow \eta_{0}\left(x_{1}^{*}\right)=\xi_{0}\left(x_{1}^{*}, \ldots, x_{k}^{*}\right)$. An easy induction shows that $\xi_{n}\left(x_{1}^{n}, \ldots, x_{k}^{n}\right) \rightarrow \xi_{0}\left(x_{1}^{*}, \ldots, x_{k}^{*}\right)$.
4. Now we shall give some remarks on subadditive functionals.

Given a positive integer $k$, we denote by $S_{k}^{*}$ the class of functionals defined in $X$ such that for arbitrary $x_{i} \in X \quad(i=1, \ldots, k+1)$ we have the inequality

$$
\xi\left(x_{1}+\ldots+x_{k+1}\right) \leqslant \xi\left(x_{1}\right)+\ldots+\xi\left(x_{k+1}\right) .
$$

Let us put $S_{k}=S_{k}^{*}-\bigcup_{i=1}^{k-1} S_{i}^{*}$.

If $\xi \epsilon S_{k}$, we say that $\xi$ is a subadditive functional of order $k$. Evidently, the classes $S_{k}^{*}$ are non-empty. We prove that $S_{k}$ are also non-empty.

4.1. If $\eta$ is an additive functional in $X$ then $\xi(x)=\mid \sin (\eta(x)+\pi / k||$ belongs to $\boldsymbol{S}_{k}$.

Indeed, since $|\sin t| \epsilon S_{1}$ we have

$$
\begin{gathered}
\xi\left(x_{1}+\ldots+x_{k+1}\right)=\mid \sin \left(\eta\left(x_{1}\right)+\ldots+\eta\left(x_{k+1}\right)+\pi / k \mid\right. \\
\left.\leqslant\left|\sin \left(\eta\left(x_{1}\right)+\pi / k\right)\right|+\ldots+\sin \left|\eta\left(x_{k+1}\right)+\pi\right| k\right)=\xi\left(x_{1}\right)+\ldots+\xi\left(x_{k+1}\right) .
\end{gathered}
$$

On the other hand, there exist $x_{1}, \ldots, x_{i} \in X$ such that the inequality

$$
\xi\left(x_{1}+\ldots+x_{i}\right) \leqslant \xi\left(x_{1}\right)+\ldots+\xi\left(x_{i}\right) \quad \text { for } \quad i=2, \ldots, k
$$

is not satisfied. It suffices to put $\eta\left(x_{j}\right)=-\pi / k$ for $j=1,2, \ldots, i$.

If $\eta$ is a linear functional, then the functional $\xi$ is continuous and uniformly bounded in $X$. On the other hand, if $\eta$ is non-continuous everywhere in $X$ (of Hamel type) then $\xi$ is non-continuous and uniformly bounded in the whole space $X$.

4.2. Now we give a method of construction for some functionals of the class $S_{k}$.

We say that the set $\sum^{(k)} \subset X$ is a semi-modulus of order $k$ if the following conditions are satisfied:

(1) $x_{1}, \ldots, x_{k+1} \in \Sigma^{(k)}$ implies $\left(x_{1}+\ldots+x_{k+1}\right) \in \sum^{(k)}$,

(2) for every $i(i=2, \ldots, k)$ there exist points $x_{1}, \ldots, x_{i} \in \sum^{(k)}$ such that $\left(x_{1}+\ldots+x_{i}\right) \notin \sum^{(k)}$.

It is easily seen that

$$
(++) \quad \xi(x)=\left\{\begin{array}{lll}
1 & \text { for } & x \notin \Sigma^{(k)} \\
0 & \text { for } & x \in \Sigma^{(k)}
\end{array}\right. \text {, }
$$

implies $\xi \epsilon \mathbb{S}_{k}$.

For a given $x_{0} \in X, x_{0} \neq 0$, write

$$
A^{(k)}=\left\{x: x=m x_{0}+x_{0} / k, m=0, \pm 1, \pm 2, \ldots\right\}
$$


and choose $\sum^{(k)}=A^{(k)}$. Then the functional defined by $(++)$ is of the class $S_{k}$. Moreover, the set of all points of discontinuity of $\xi$ is exactly countable.

4.3. Functionals of the class $S_{k}$ may also be constructed by applying infinite series (for the case $k=1$, see [5], p. 137).

We denote by $\sum_{n}^{(k)}$ for $n=1,2, \ldots$ a semi-modulus of order $k$ such that for each positive integer $N$ and $i=2, \ldots, l_{0}$ there exist points

such that

$$
x_{1}(N, i), \ldots, x_{i}(N, i) \in \bigcap_{n=1}^{N} \sum_{n}^{(k)}
$$

$$
\left(\dot{x}_{1}(N, i)+\ldots+x_{i}(N, i)\right) \notin \bigcup_{n=1}^{N} \sum_{n}^{(k)} \text {. }
$$

Let us replace in $(++) \sum^{(k)}$ by $\sum_{n}^{(k)}$ and $\xi$ by $\xi_{n}$. Further, let the series $\left|a_{1}\right|+\left|a_{2}\right|+\ldots$ be convergent, where $\left|a_{n}\right|>0$. Then the functional

is of the class $S_{k}$.

$$
\xi(x)=\sum_{n=1}^{\infty}\left|a_{n}\right| \xi_{n}(x)
$$

Evidently, $\xi \in S_{k}^{*}$. Now we prove that for $i=2, \ldots, k$ there exist $x_{1}, \ldots, x_{i}$ such that the inequality

$$
\xi\left(x_{1}+\ldots+x_{i}\right) \leqslant \xi\left(x_{1}\right)+\ldots+\xi\left(x_{i}\right)
$$

is not satisfied. The inequality

implies

$$
\sum_{n=1}^{\infty}\left|a_{n}\right| \xi_{n}\left(x_{1}+\ldots+x_{i}\right) \leqslant \sum_{n=1}^{\infty}\left|a_{n}\right|\left(\xi_{n}\left(x_{1}\right)+\ldots+\xi_{n}\left(x_{i}\right)\right)
$$

$$
\begin{aligned}
\sum_{n=1}^{N}\left|a_{n}\right|+\sum_{n=N+1}^{\infty}\left|a_{n}\right| \xi_{n}\left(x_{1}(N, i)+\ldots+x_{i}(N, i)\right) & \\
& \leqslant \sum_{n=N+1}^{\infty}\left|a_{n}\right|\left(\xi_{n}\left(x_{1}(N, i)\right)+\ldots+\xi_{n}\left(x_{i}(N, i)\right)\right)
\end{aligned}
$$

for $i=2, \ldots, k$. Thus for sufficiently large $N$ the last inequality is not satisfied for $i=2, \ldots, k$. Hence $\xi \in S_{k}$.

4.31. Let $\xi \in S_{k}^{*}$. Every functional of the form $\xi(x)+c \xi\left(x_{0}\right)$, where $c \geqslant\left[(k-1) / 2-\left(1+(-1)^{k}\right) / 4\right]$ and $\xi\left(x_{0}\right)=\xi\left(-x_{0}\right) \geqslant 0$ is of the class $\left.\left.S_{(3+(-1)}^{*}\right)^{k}\right) / 2$. Moreover, since $\xi(0) \geqslant 0$, the functional $\xi(x)+c \xi(0)$ is of the class $S_{1}$ for every $c \geqslant(k-1) / 2$.

These remarks immediately follow from $(+)$.

4.32. If $\xi \in S_{k}$ then $\xi \in S_{k i l}^{*}$ for $n=1,2, \ldots$
4.33. Suppose that for $\xi \in S_{k_{0}}^{*}$ there exists a point $x_{0} \in X$ such that $\xi$ is continuous at $x_{0}$ and $\xi\left(x_{0}\right)+\xi\left(-x_{0}\right)=0$. Then $\xi$ is continuous in the whote space $X$.

Since $\xi \in S_{k}$, we have

and

$$
\xi(x+h) \leqslant \xi(x)+k\left(\xi\left(x_{0}+h / k\right)+\xi\left(-x_{0}\right)\right)
$$

Hence

$$
\xi(x) \leqslant \xi(x+h)+k\left(\xi\left(x_{0}-h / k\right)+\xi\left(-x_{0}\right)\right) \text {. }
$$

$|\xi(x)-\xi(x+h)| \leqslant k \max \left(\xi\left(x_{0}+h / k\right)+\xi\left(-x_{0}\right), \xi\left(x_{0}-h / k\right)+\xi\left(-x_{0}\right)\right)$ for an arbitrary point $x \in \bar{X}$.

4.34. If $\xi \in S_{k}$ and $\xi(x)=\xi(-x)$ for every $x \in X$, then $\left.\xi \geqslant 0^{2}\right)$.

It suffices to put in $(+) x_{i}=(-1)^{i} x$ for $i=1, \ldots, k+1$.

4.4. Let $\xi_{n}$ be a functional continuous on $X$ for $n=1,2, \ldots$ and let $\xi_{n} \in S_{k}^{*}$ for every n. Further, let the sequence $\left\{\xi_{n}\right\}$ be bounded above in a CRB of a sphere in $X$. Then there exists in $X$ a sphere $K\left(x_{0}, r\right)$ such that the sequence $\left\{\xi_{n}\right\}$ is uniformly bounded above in $K\left(x_{0}, r\right)$.

We denote by $R$ the set of all points $x$ such that the sequence $\left\{\xi_{n}\right\}$ is bounded above at $x$. Let us observe that there exist rational numbers $\beta_{1} \geqslant 0, \ldots, \beta_{m} \geqslant 0$, satisfying the inequality $\beta_{1}+\ldots+\beta_{m} \leqslant 1$ and such that the set

$$
E=\left\{x: x=\sum_{i=1}^{m} \beta_{i}\left(x_{i}-a\right)+a, x_{i} \in B^{*}\right\}
$$

is of the second category in $X$ (compare the proof in section 2). We choose such a positive integer $p$, which is a multiplicity of $k$, that the numbers $p \beta_{i}$ are non-negative integers. The set $E_{p}=\{y: y=p x+a, x \in E\}$ is of the second category. Since $x \in B^{*}$ implies $\xi_{n}(x)<G(x)$ for $n=1,2, \ldots$, we have for $y \in E_{p}$ and $n=1,2, \ldots$

$$
\begin{aligned}
\xi_{n}(y) & =\xi(p x+a)=\xi_{n}\left(\sum_{i=1}^{m} p \beta_{i} x_{i}+p\left(1-\sum_{i=1}^{m} \beta_{i}\right) a+a\right) \\
& \leqslant \sum_{i=1}^{m} p \beta_{i} \xi_{n}\left(x_{i}\right)+p\left(1-\sum_{i=1}^{m} \beta_{i}\right) \xi_{n}(a)+\xi_{n}(a) \\
& <(1+p) \max \left(G\left(x_{1}\right), \ldots, G\left(x_{m}\right), G(a)\right)=G(y) .
\end{aligned}
$$

Thus $y \in R$. The set $R$ is an $F_{\sigma}$ and of the second category. Hence there exists a sphere $K\left(x_{0}, r\right) \subset R$ such that the sequence $\left\{\xi_{n}\right\}$ is uniformly bounded above in $K\left(x_{0}, r\right)$.

2) The inequality $\xi \geqslant \eta$ for two functionals $\xi, \eta$ means $\xi(x) \geqslant \eta(x)$ for arbitrary $x \in X$. 
5. Let $\xi_{n}$ be a functional continuous on $X$ for $n=1,2, \ldots$, and let $\xi_{n} \epsilon S_{k}^{*}$ for every $n$. Further, let the sequence $\left\{\xi_{n}\right\}$ be bounded above in a set dense in $X$ and in a CRB of a sphere in $X$. Then the sequence $\left\{\xi_{n}\right\}$ is uniformly bounded above in every bounded set.

According to 4.4 there exists a sphere $K\left(x_{0}, r\right)$ such that $\xi_{n}(x)<G$ for $n=1,2, \ldots, x \in K\left(x_{0}, r\right)$. We choose a point $x_{1} \in K\left(x_{0}, r\right)$ such that there exists a constant $G\left(-x_{1}\right)$ such that $\xi_{n}\left(-x_{1}\right)<G\left(-x_{1}\right)$ for $n=1,2, \ldots$ Further we choose a sphere $K\left(x_{1}, r_{1}\right) \subset K\left(x_{0}, r\right)$. If the set $A$ is bounded, then the set $\left\{z: z=y-x_{1}, y \in A\right\}$ is bounded too. We observe that there exists such a positive integer $p$, which is a multiplicity of $k$, that there exists for every $y \in A$ a point $x \in K\left(x_{1}, r_{1}\right)$ such that $y=p\left(x-x_{1}\right)+x_{2}$. Thus we have for $y \in A$

$$
\begin{aligned}
\xi_{n}(y)=\xi_{n}\left(p\left(x-x_{1}\right)+x_{1}\right) & \leqslant p \xi_{n}(x)+p \xi_{n}\left(-x_{1}\right)+\xi_{n}\left(x_{1}\right) \\
& <(2 p+1) \max \left(G, G\left(-x_{1}\right)\right) .
\end{aligned}
$$

5.1. From 4.4 we conclude the following:

Suppose the functional $\xi_{n}$ to be continuous on $X, \xi_{n} \in S_{k}^{*}$ and $\xi_{n} \leqslant \xi_{n+1}$ for $n=1,2, \ldots$ If the sequence $\left\{\xi_{n}\right\}$ is convergent in a CRB of a sphere in $X$, then there exists a sphere $K\left(x_{0}, r\right) \subset X$ such that $\left\{\xi_{n}\right\}$ is convergent in $K\left(x_{0}, r\right)$.

5.2. From 5 we conclude the following:

Let $\xi_{i b}$ be a functional continuous on $X$ for $n=1,2, \ldots$, and let $\xi_{n} \in S_{k}^{*}$, $\xi_{n} \leqslant \xi_{n+1}$ for every $n$. If the sequence $\left\{\xi_{n}\right\}$ is convergent in a set dense in $X$ and in a CRB of a sphere in $X$, then it is convergent in the whole space $X$. holds:

. The method of proving 4.4 and 5 shows that the following theorem

Suppose the functional $\xi_{n}$ to be continuous on $X, \xi_{n} \in S_{k}^{*}$ and $\xi_{n}(x)$ $=\xi_{n}(-x)$, for $n=1,2, \ldots$, and $x \in X$. Further let the sequence $\left\{\xi_{n}\right\}$ be bounded above in an LRB of a sphere in $X$. Then the sequence $\left\{\xi_{n}\right\}$ is uniformly bounded in every bounded set in $X$.

6.1. Theorem 6 implies the following:

Let $\xi_{n}$ be a functional continuous on $X$ for $\dot{n}=1,2, \ldots$ and let $\xi_{n} \in \boldsymbol{S}_{k}^{*}$, $\xi_{n} \leqslant \xi_{n+1}$ and $\xi_{n}(x)=\xi_{n}(-x)$ for every $n$ and $x \in X$. Further suppose the sequence $\left\{\xi_{n}\right\}$ to be convergent in an $L R B$ of a sphere in $X$. Then the sequence $\left\{\xi_{n}\right\}$ is convergent in the whole space $X$.

6.2. Let us suppose the functional $\xi_{n}$ to be continuous on $X, \xi_{n} \geqslant 0$ and $\xi_{n} \in S_{k}^{*}$ for $n=1,2, \ldots$ Moreover, let $\xi_{n}(x) \rightarrow 0$ in a CRB of a sphere in $X$. Then $\xi_{n}(x) \rightarrow 0$ in a sphere in $X$.

Denote by $E$ the set of all points $x \in X$ such that $\xi_{n}(x) \rightarrow 0$. Arguments analogical to that used in 4.4 show that the set $E$ is of second category.
Since $E$ is $F_{\sigma \delta}, 7.1$ implies that the set $E^{\prime}=\left\{x: x=\left(x_{1}+x_{2}\right) / 2, x_{1}, x_{2} \in E\right\}$ contains a sphere. Hence the set $E^{\prime \prime}=\left\{y: y=2 k x+a, x \in E^{\prime}\right\}$, where $a \epsilon B^{*}$, also contains a sphere. The inequality

implies $E^{\prime \prime} \subset E$.

$$
\xi_{n}(y) \leqslant k \xi_{n}\left(x_{1}\right)+k \xi_{n}\left(x_{2}\right)+\xi_{n}(a)
$$

6.3. Suppose the functional $\xi_{n}$ to be continuous on $X, \xi_{n} \in S_{k}^{*}$ and $\xi_{n}(x)=\xi_{n}(-x)$ for $n=1,2, \ldots, x \in X$. Further $\xi_{n}(x) \rightarrow 0$ in an LRB of a sphere in $X$. Then $\xi_{n}(x) \rightarrow 0$ everywhere.

Arguments analogical to that. of 6.2 show that $\xi_{n}(x) \rightarrow 0$ in a sphere $K\left(x_{0}, r\right)$. Applying the condition $\xi_{n}(x)=\xi_{n}(-x)$ we use the arguments of 5 with $x_{0}=x_{1}$.

6.31. We observe that the lemmas, theorems and corollaries 4.4 , $5,5.1,5.2,5.3,6,6.1,6.2$ and 6.3 remain true if we replace the assumption of the continuity of the functional $\xi_{n}$ for $n=1,2, \ldots$, by the lower semi-continuity.

6.4. Let the functional $\xi_{n}$ be continuous on $X$ for $n=1,2, \ldots$ and let $\xi_{n} \in S_{k}^{*}, \xi_{n}(x)=\xi_{n}(-x)$ for every $n$ and $x \in X$. If $\xi_{n}(x) \rightarrow 0$ in the whole space $X$, then the $\xi_{n}$ are equicontinuous at each point of the space $X$.

First we prove the equicontinuity of $\xi_{n}$ at zero. Choose an arbitrary $\varepsilon>0$ and write $E_{N}=\left\{x: \xi_{n}(x) \leqslant \varepsilon / 4 k, n \geqslant N\right\}$. Since the set $E_{N}$ is closed, there exists $N_{0}$ such that $E_{N_{0}}$ contains a sphere $K\left(x_{0}, \delta_{0}\right)$. Let $h / k \in K\left(0, \delta_{0}\right)$. Since $h=k\left(h / k+x_{0}\right)+k\left(-x_{0}\right)+1 \cdot 0$ and $n \geqslant N_{1}$ implies $\xi_{n}(0)<\varepsilon / \mathbf{4}$, we obtain the inequalities

$$
\left|\xi_{n}(0)-\xi_{n}(h)\right| \leqslant k \xi_{n}\left(\frac{h}{k}+x_{0}\right)+k \xi_{n}\left(x_{0}\right)+2 \xi_{n}(0)<\varepsilon
$$

for $n \geqslant N=\max \left(N_{0}, N_{1}\right),\|h / k\|<\delta_{0}$. Hence it follows that, for sufficiently small $\delta>0,\|h\|<\delta$ implies. $\left|\xi_{n}(0)-\xi_{\eta}(h)\right|<\varepsilon$ for $n=1,2, \ldots$ Applying the inequality

$$
\left|\xi_{n}(x)-\xi_{n}(x+h)\right| \leqslant k \xi_{n}\left(\frac{h}{k}\right),
$$

valid for arbitrary $x \epsilon X$, we obtain the equicontinuity of $\xi_{n}$ at each point of the space $X$.

6.41. Our proofs of the lemmas and theorems on subadditive functionals of order $k$ may be applied to $(F)$-spaces without any change.

6.42. Let $U_{n}$ denote linear operations from $X$ to $Y$ for $n=1,2, \ldots$, $X, Y$ being $(F)$-spaces. We shall prove a known theorem (see [7]):

If the sequence $\left\{U_{n}\right\}$ is bounded at each point of $X$ then $U_{n}$ are equicontinuous at each point of $X$. 
Since $t_{n} \rightarrow 0$ implies $U_{n}\left(t_{n} x\right) \rightarrow 0$ for arbitrary $x \in X$, we have $\xi_{n}(x)$ $=\left\|U_{n}(x)\right\| \rightarrow 0$ for every $x \in X$. It follows from 6.4 that $\xi_{n}$ are equicontinuous at every point $x \in X$. Hence $x_{n} \rightarrow 0$ implies $U_{n}\left(t_{n} x_{n}\right) \rightarrow 0$, e. g., for every $\varepsilon>0$ there exists a number $\delta>0$ such that the inequalities $|t| \leqslant \delta$ and $\|x\|<\delta$ imply $\left\|U_{n}(t x)\right\|<\varepsilon$ for $n=1,2, \ldots$ Since, for sufficiently small $\eta>0,\|x\|<\eta$ implies $\|x / \delta\|<\delta,\|x\|<\eta$ implies $\left\|U_{n}(x)\right\|<\varepsilon$ for $n=1,2, \ldots$

6.43. Let $U_{n}$ be a linear operation from $X$ to $Y$ for $n=1,2, \ldots$, $X, Y$ being $(F)$-spaces. Applying 6.3 we may formulate a well known theorem (see [ $\tau]$ ) in the following generalized form:

The set of the points of boundedness of the sequence $\left\{U_{n}\right\}$ is either identical with the whole space or it is not an LRB of any sphere.

6.44. There exists a sequence $\left\{\xi_{n}\right\}$ of functionals continuous on $X$ and of the class $S_{k}$, such that $\left\{\xi_{n}\right\}$ is convergent to a non-continuous functional $\xi_{0} \in S_{k}$. (Consequently, there exists a sequence $\left\{\xi_{n}\right\}$ of continuous functionals of the class $S_{k}$ convergent in the whole of space and such that the functionals $\xi_{n}$ are not equicontinuous for a point $x \in X$ ).

We define the functionals $\xi_{n}$ as follows:

$$
\xi_{n}(x)=\sqrt[n]{\sin (\eta(x)+\pi / k)} \quad \text { for } \quad n=1,2, \ldots, x \in X,
$$

where $\eta$ is a linear functional in $X$ which vanishes only at zero. It is easily seen that $\xi_{n} \in S_{k}$ for every $n$ (compare 4.1) and that the set of all zeros of the functional $\xi_{n}$ is identical with a semi-modulus of order $k$; it may be denoted by $\Sigma^{(k)}$. This set does not depend on $n$. Functionals $\xi_{n}$ tend to the functional $\xi_{0}$ given by $(++)$ and $\Sigma^{(k)}$ in 4.2 .

We shall return to the investigations of the general properties of convex functionals and subadditive functionals of order $k_{i}$ in another paper.

7. Now we give some examples related to the previous theorems.

7.1. If the set $E \subset X$ is of the second category and satisfies the Baire condition, then the set $\left\{x: x=\left(x_{1}+x_{2} / 2, x_{1}, x_{2} \in E\right\}\right.$ contains a sphere.

Since the set satisfies the Baire condition, there exist a point $a \in E$ and a sphere $K(a, \varrho)$ such that the set $E \cap K(a, \varrho)$ is residual in $K(a, \varrho)$ (see [2]). Let us consider an arbitrary point $x \in \mathcal{K}(a, \varrho)$ and write $E(x)=\{y: 2 x-y \in E\}$. Notice that $E \cap E(x) \neq 0$. Thus $x \in K(a, \varrho)$ implies $x=\left(x_{1}+x_{2}\right) / 2$ with $x_{1}, x_{2} \in E$.

7.2. If $E \subset A \subset X, A$ being a bounded set, and if $E$ is of second category and satisfies the Baire condition, then $E$ is a ORB of $A$.
According to 7.1. there exists a sphere $K(a, \varrho)$ such that $a \in E$ and $x \in K(a, \varrho)$ implies $x=\left(x_{1}+x_{2}\right) / 2$, where $x_{1}, x_{2} \in E$. Since the set $A$ is bounded, there exists a rational number $\beta \geqslant 0$ such that $y \in A$ implies $y=\beta(x-a)+a$, where $x \in K(a, \varrho)$, e. $g ., y \in A$ implies $y=\beta\left(x_{1}-a\right) / 2+$ $+\beta\left(x_{2}-a\right) / 2+a$, where $x_{1}, x_{2} \in E$.

The method of our proof shows that 7.1 and 7.2 remain true for (F)-spaces.

7.21. From 2 and 7.2 we conclude the following:

Let the functional $\xi$ defined and convex in a convex region $D$ be uniformly bounded above in a subset of the second category satisfying the Baire condition. Then $\xi$ is continuous in $D$.

7.22. From 7.21 we conclude the following:

If the functional $\xi$ defined and convex in a convex region $D$ satisfies the Baire condition, it is continuous in $D$.

7.23. Theorem 7.1 and the arguments of $2,3.1,1.3$ show that 7.21 and 7.22 remain true if the space $X$ is of type $(F)$.

We say that the set $E^{*} \subset \bar{X}$ is homothetic with the set $E$ if $E^{*}=\{y: y=\tau x+b, x \epsilon E\}$, where $b \in X$ and $\tau$ is a real number. Denote by $C$ the Cantor ternary set. Put $\Gamma=\{x:\|x\|=t, x \in X, t \in C\}$. The set $\Gamma$ is non-dense in $X$.

7.3. If the set $\Gamma^{*} \subset K\left(x_{0}, r\right)$ is homothetic with the set $\Gamma$ then $\Gamma^{*}$ is a $C R B$ of the sphere $K\left(x_{0}, r\right)$.

We conclude from the triadic expansion of numbers of the closed interval $\langle 0,1\rangle$ and from the definition of the set $C$ that $t \epsilon\langle 0,1\rangle$ implies $t=\left(t_{1}+t_{2}\right) / 2$, where $t_{1}, t_{2} \epsilon O$. Hence it immediately follows that $\Gamma^{*}$ is a CRB of $K\left(x_{0}, r\right)$.

7.31. We conclude from 7.3 that in the formulation of 7.21 the set of the second category satisfying the Baire condition can be replaced by $\Gamma^{*}$.

7.4. Further we denote by $E_{n}$ the $n$-dimensional real Euclidean space. We define in $E_{n}$ the norm $\|x\|=\max \left(\left|x_{1}\right|, \ldots,\left|x_{n}\right|\right)$, where $x=\left(x_{1}, \ldots, x_{n}\right)$. In the following theorems the notion of measurability will be understood in the sense of Lebesgue. We obtain the following result, analogical to 7.1 :

If the measurable set $E \subset E_{n}$ is of finite positive measure then the set $\left\{x: x=\left(x_{1}+x_{2}\right) / 2, x_{1}, x_{2} \in E\right\}$ contains a sphere.

Let us observe that there exists for every $1 \geqslant \alpha>\frac{1}{2}$ a point $a \in E$ and a real number $\varrho>0$ such that $\mu(E \cap K(a, \varrho))=\alpha \mu(E)$ where $\mu(E)$ denotes the measure of the set $E$. Put $\beta=1-\sqrt[n]{\frac{3}{2}-\alpha}$. For each $x \in K(a, \beta 2)$ 
we write $E^{*}(x)=\left\{y: 2 x-y \in E^{*}\right\}$; where $E^{*}=E \cap K(a, \varrho)$ and $K(x)=$ $=\{y: 2 x-y \in K(a, \varrho)\}$.

Since

$$
\begin{aligned}
\mu\left(E^{*} \cap K(x)\right) & \geqslant \mu\left(E^{*}\right)-\mu(K(a, \varrho)-K(x)) \\
& =\mu\left(E^{*}\right)-\mu(K(a, \varrho))+\mu(K(a, \varrho) \cap K(x)) \\
& >\mu\left(E^{*}\right)-\mu(K(a, \varrho))+(2 r(1-\beta))^{n}=(2 r)^{n} / 2
\end{aligned}
$$

and similarly

we have

$$
\mu\left(E^{*}(x) \cap K(a, \varrho)\right)>(2 r)^{n} / 2
$$

$$
\begin{aligned}
\mu\left(E^{*} \cap E^{*}(x)\right) \geqslant \mu\left(E^{*} \cap E^{*}(x)\right)-\mu(K(a, \varrho) \frown K(x))+ \\
\quad+\mu\left(\left(E^{*} \cup E^{*}(x)\right) \cap(K(a, \varrho) \cap K(x))\right) \\
=\mu\left(E^{*}(x) \frown K(a, \varrho)\right)+\mu\left(E^{*} \cap K(x)\right)- \\
\quad-\mu(K(a, \varrho) \frown K(x))>(2 r)^{n}-(2 r)^{n}=0 .
\end{aligned}
$$

Since $E^{*} \cap E^{*}(x) \neq 0$, we have proved that $x \in K(a, \varrho)$ implies $x=\left(x_{1}+x_{2}\right) / 2$, where $x_{1}, x_{2} \in E$.

7.5. If we apply 7.4, arguments similar to that of 7.2 show that:

An arbitrary measurable set $E \subset E_{n}$ of positive measure contained. in a sphere $K\left(x_{0}, r\right)$ is a CRB of this sphere.

7.51. It follows from 2 and 7.5 that:

If the function $f\left(x_{1}, \ldots, x_{n}\right)$ of $n$ variables, defined and convex in a convex set $D \subset E_{n}$, is. uniformly bounded above in a set $E \subset D$ of positive measure then $f\left(x_{1}, \ldots, x_{n}\right)$ is continuous in $D$.

Especially, we obtain for $n=1$ the theorem given by A. Ostrowski (see [11]).

7.52. We conclude from 7.51 that: in $D$.

If the function defined in 7.51 is measurable in $D$ then it is continuous

For $n=1$ we obtain the theorem of W. Sierpiński (see [12]).

7.53. It follows from 7.52 especially that:

If a function $f\left(x_{1}, \ldots, x_{n}\right)$ defined and additive in $E_{n}$ is measurable in $E_{n}$ then it is linear $\left.{ }^{3}\right)$.

7.54. We conclude from 7.3 that:

There exist sets of measure zero contained in a sphere $K\left(x_{0}, r\right)$, which are $C R B$ of this sphere.

7.6. We say that the set $E \subset X$ is totally asymmetric if there exists for every $x_{0} \in E$ a sphere $K\left(x_{0}, r\right)$ such that $x \in E \cap K\left(x_{0}, r\right)$ implies $2 x_{0}-x \notin E$.

$\left.{ }^{3}\right)$ In the case $n=1$ Theorem 7.53 was first proved by M. Fréchet. This theorem has been the object of many proofs, see for example [1] and [13].
7.61. Since in the proof of $\pi .1$ the point $a$ is taken from the set $E$, we find that:

If the space $X$ is an $(F)$-space then every set satisfying the Baire condition and contained in a totally asymmetric set is of the first category.

In the case $X=E_{1}$ we obtain the theorem given by $\mathrm{S}$. Marcus (see [6]).

7.62. As in 7.61 we find from 7.4 that:

Every measurable set in $E_{n}$ contained in a totally asymmetric set is of measure zero.

Especially we obtain for $n=1$ a theorem given by $\mathbf{S}$. Marcus (see [6]).

7.7. Let $\varphi(x)$ denote a function defined in $E_{1}$, periodic with the period $l$ and integrable in $(0, l)$. If $\left|\lambda_{n}\right| \rightarrow \infty$ then for arbitrary $x_{0} \in E_{1}$ and $h>0$ we have the relation

$$
\lim _{n \rightarrow \infty} \frac{1}{2 h} \int_{x_{0}-\hbar}^{x_{0}+h}\left|\varphi\left(\lambda_{n} t\right)\right| d t=\frac{1}{l} \int_{0}^{l}|\varphi(t)| d t .
$$

7.8. Let $[x]$ denote the greatest integer $\leqslant x$. Let us observe that the function $\xi(x)=x+1 / k-[x+1 / k]$ is of the class $S_{k}$; it may be obtained by arguments similar to that of 4.1 .

8. If the series

$$
\sum_{n=1}^{\infty}\left|a_{n}\right|\left(\lambda_{n} x+\frac{1}{k}-\left[\lambda_{n}+\frac{1}{k}\right]\right), \quad\left|\lambda_{n}\right| \rightarrow \infty,
$$

is convergent in $a C R B$ of an interval $(\alpha, \beta)$ then

$$
\sum_{n=1}^{\infty}\left|a_{n}\right|<\infty
$$

Write

$$
\xi_{n}(x)=\sum_{i=1}^{n}\left|a_{i}\right|\left(\lambda_{i} x+\frac{1}{k}-\left[\lambda_{i} x+\frac{1}{k}\right]\right) .
$$

Evidently $\xi_{n} \in S_{k}^{*}$ and $\xi_{n}$ is lower semi-continuous for $n=1,2, \ldots$ Since the sequence $\left\{\xi_{n}\right\}$ is convergent in CRB of the interval $(\alpha, \beta)$, according to 4.4 and 6.31 , it is uniformly bounded above in an interval $\left(x_{0}-h, x_{0}+h\right)$. Applying 7.7 we obtain the convergence of the series

$$
\sum_{n=1}^{\infty}\left|a_{n}\right| \text {. }
$$


8.1. If the series.

$$
\sum_{n=1}^{\infty}\left(\left|a_{n} \cos \lambda_{n} x\right|+\mid b_{n} \sin \lambda_{n} x\right), \quad\left|\lambda_{n}\right| \rightarrow \infty
$$

is convergent in an $L R B$ of an interval $(\alpha, \beta)$ then

$$
\sum_{n=1}^{\infty}\left(\left|a_{n}\right|+\left|b_{n}\right|\right)<\infty .
$$

Put

$$
\xi_{n}(x)=\sum_{i=1}^{n}\left(\left|a_{i} \cos \lambda_{i} x\right|+\left|b_{i} \sin \lambda_{i} x\right|\right) .
$$

The functional $\xi_{n}$ satisfies the assumptions of the section 6 for $n=1,2, \ldots$ Hence the sequence $\left\{\xi_{n}\right\}$ is uniformly bounded in an interval $\left(x_{0}-h, x_{0}+h\right)$. If we apply 7.7 as above, we obtain the convergence of the series

$$
\sum_{n=1}^{\infty}\left(\left|a_{n}\right|+\left|b_{n}\right|\right) \text {. }
$$

8.11. A certain modification of the known method of proof yields, if we apply 8.1 and 7.7 (compare [14], p. 133), a more general result ${ }^{ \pm}$):

If the series

$$
\sum_{n=1}^{\infty}\left|a_{n} \cos \lambda_{n} x+b_{n} \sin \lambda_{n} x\right|, \quad\left|\lambda_{n}\right| \rightarrow \infty,
$$

is convergent in an LRB of an interval $(\alpha, \beta)$ then

$$
\sum_{n=1}^{\infty}\left(\left|a_{n}\right|+\left|b_{n}\right|\right)<\infty
$$

It may be pointed out that the results 8.11 and 8.1 are obtained without the use of the Lebesgue integral.

8.2. Let $\eta_{n}$ be a linear functional defined in $X$ for $n=1,2, \ldots$ and let $\left\|\eta_{n}\right\| \rightarrow \infty$. If

in $L R B$ of a sphere, then

$$
\left(a_{n} \cos \eta_{n}(x)+b_{n} \sin \eta_{n}(x)\right) \rightarrow 0
$$

$$
\left(\left|a_{n}\right|+\left|b_{n}\right|\right) \rightarrow 0
$$

Write $\xi_{n}(x)=a_{n} \cos \eta_{n}(x)+b_{n} \sin \eta_{n}(x)$ and $\xi_{n}^{*}(x)=b_{n} \cos \eta_{n}(x)-$ $-a_{n} \sin \eta_{n}(x)$. If we put $x=z+y$, where $x, y, z \epsilon$, , then we obtain

$$
\xi_{n}(x)=\xi_{n}(z) \cos \eta_{n}(y)+\xi_{n}^{*}(z) \sin \eta_{n}(y) .
$$

4) Special classes LRB were used in considering absolute convergence of trigonometrical series by W. Niemytzki in [8].
Let $z$ be a fixed point of $B$. Our assumptions imply $\xi_{n}(z) \rightarrow 0$. Since $\xi_{n}(x) \rightarrow 0$ for $x \in B$, we have $\xi_{n}^{*}(z) \sin \eta_{n}(y) \rightarrow 0$ for $y \in B(z)$, where $B(z)=$ $=\{y: y=x-z, x \in B\}$. The set $B(z)$ is an LRB of a sphere. Since the sequence $\left\{\left|\xi_{n}^{* *}\right|\right\}$, where $\xi_{n}^{* *}(y)=\xi_{n}^{*}(z) \sin \eta_{n}(y)$, satisfies the assumption of 6.3 , we obtain $\xi_{n}^{* *}(y) \rightarrow 0$ for every $y \in X$. Hence it follows that $\xi_{n}(x) \rightarrow 0$ for every $x \epsilon X$. If we put $x=0$ we obtain $a_{n} \rightarrow 0$. Write $\xi_{n}^{\prime}(x)=$ $=\left|b_{n} \sin \eta_{n}(x)\right|$. Then the sequence $\left\{\xi_{n}^{\prime}\right\}$ satisfies the assumptions of 6.3 . Hence $\xi_{n}^{\prime}(x) \rightarrow 0$ for every $x \in X$. According to 6.4 the functionals $\xi_{n}^{\prime}$ are equicontinuous at the point $x=0$. If we put $x_{n}=y_{n} /\left\|\eta_{n}\right\|$, where $\left|\eta_{n}\left(y_{n}\right)\right| \geqslant\left\|\eta_{n}\right\| / 2$ and $\left\|y_{n}\right\|=1$, then we obtain $1 \geqslant\left|\eta_{n}\left(x_{n}\right)\right| \geqslant 1 / 2$. Since $\xi_{n}^{\prime}\left(x_{n}\right)=\left|b_{n} \sin \eta_{n}\left(x_{n}\right)\right| \rightarrow 0$, we have $b_{n} \rightarrow 0$.

8.3. Denote by $\left\{\vartheta_{n}\right\}$ an arbitrary sequence of real numbers. Retaining the notation of 8.2 we obtain the following theorem:

The set

$E(\vartheta)=\left\{x:\left(\eta_{n}(x)+\vartheta_{n}-\left[\eta_{n}(x)+\vartheta_{n}\right]\right) \rightarrow \vartheta,\left\|\eta_{n}\right\| \rightarrow \infty\right\}$, where $0 \leqslant \vartheta \leqslant 1$, is not an LRB of any sphere.

Suppose that $E(\vartheta)$ is an LRB of a sphere. Then $\sin \pi\left(\eta_{n}(x)+\vartheta_{n}-\vartheta\right)$ $\rightarrow 0$ in this LRB, which is contrary to 8.2 .

8.31. From 8.3 we immediately conclude the following:

Let the function $\varphi(x)$ periodic with period 1 be continuous and let $\varphi(x)$ vanish only at $\vartheta$ in the interval $(0,1)$, where $0 \leqslant \vartheta \leqslant 1$. If the sequence $\left\{a_{n} \varphi\left(\eta_{n}(x)+\vartheta_{n}\right)\right\}$ is convergent to zero in an LRB of a sphere then $a_{n} \rightarrow 0$ (compare [9]).

9. Finally we give an application to the theory of $\left(F^{\prime}\right)$-spaces. In connexion with other applications of subadditive functionals concerning the theory of $(F)$-spaces we refer the reader to the paper [10].

Let $X$ be the $(F)$-space under the norm \|| || satisfying for a number $\alpha>0$ the condition

$$
\sup _{\mid t_{i}>0} \frac{\|t x\|}{|t|^{a}}<\infty \quad \text { for } \quad x \in X .
$$

Then the norm \|\|$^{*}$ where

$$
\|x\|^{*}=\sup _{|t|>0} \frac{\|t x\|}{|t|^{a}}
$$

is equivalent to the norm \|\| and satisfies the condition $\|t x\|^{*}=|t|^{\alpha}\|x\|^{*}$.

It follows from the inequality $\|x\|^{*} \geqslant\|x\|$ that convergence with respect to the norm \|\|$^{*}$, implies convergence with respect to the norm \|\| . Let $\left\{t_{n}\right\}$ denote the sequence of all rational numbers different from 
zero. Put $\xi_{n}(x)=\left\|\left.\left|t_{n} x \| /\right| t_{n}\right|^{\alpha}\right.$ for $n=1,2, \ldots$ The functionals $\xi_{n}$ satisfy the assumptions of 6 . Thus there exists a constant $G$ such that $\xi_{n}(x)<G$ for $x \in K(0,1)$. The continuity of the norm \|\| implies $\|t x\| /|t|^{a}<G$ for real $t$ and for $x \in K(0,1)$. Let $\varepsilon$ be an arbitrary positive number. We choose a number $\tau>0$ such that $G<\tau^{\alpha} \varepsilon$. There exists a $\delta>0$ such that $\|x\|<\delta$ implies $\|\tau x\|<1$. Consequently, the inequality $\|t \tau x\| /|t|^{a}<G$ is satisfied. Hence $\|x\|<\delta$ implies $\|x\|^{*}<\varepsilon$

\section{References}

[1] A. Alexiewicz et W. Orlicz, Remarque sur l'équation fonctionnelle $f(x+y)=f(x)+f(y)$, Fund. Math. $33(1945)$, p. 314-315.

[2] S. Banach, Théorème sur les ensembles de première oatégorie, ibidem 16 1930), p. $395 \cdot 398$.

[3] - Théorie des opérations linéaires, Warszawa 1932.

[4] G. H. Hardy, J. E. Littlewood and G. Pólya, Inequalities, Cambridge 1934.

[5] E. Hille, Funetional analysis and semigroups, Now York 1948.

[6] S. Marcus, Sur un problème de la théorie de la mesure de H. Steinhaus et S. Ruziewicz, Bull. Acad. Pol. Sei., Cl. III, 4 (1956), p. 197 - 199.

[7] S. Mazur und W. Orlicz, Über Folgen linearer Operationen, Studia Math. 4. (1933), p. $152-157$.

[8] В. Н емыцкий, O некоторых классах линейных множеств в связии с абсолютной сходимостью тригонометрических рлдов, Мат. сборник 33 (1926), p. 5 - 32 .

[9] W. Orlicz, Une généralisation d'un théorème de Cantor-Lebesgue, Annales Soc. Pol. Math. 21 (1948), p. 38-45.

[10] - Linear operations in Saks spaees (II), Studia Math. 15 (1956), p. $38-45$.

[11] A. Ostrowski, Über die F'unktionalgleichung der Exponentialfunktion und verwandte Funktionalgleichungen, Jahresbericht der Deutschen Mathematiker Vereinigung 38 (1929), p. 54-62.

[12] W. Sierpiński, Sur les fonctions convexes mesurables, Fund. Math. 1 (1920), p. $125-129$.

[13] - Sur l'équation fonotionnelle $f(x+y)=f(x)+f(y)$, ibidem 1 (1920), p. $116 \cdot 122$.

[14] A. Zygmund, Trigonometrical series, Warszawa 1935.

\section{INSTYTUT MATEMATYCZNY POLSKIET AKADTMTI NAUK}

MATHEMATICAL INSTITUTE OF THE POLISH ACADEMY OF SCIENCRS

Ręu par la Rédaction le 8. 12.1956

Problème de l'analyticité par rapport à un opérateur linéaire*

par

M. NICOLESCU (Bucarest)

1. Définitions et premier axiome. Cas intermédiaires. Ce qui suit constitue un schéma général abstrait de plusieurs résultats obtenus par l'auteur dans la théorie des fonctions polyharmoniques, ou dans la théorie de l'opérateur hyperbolique itéré ou encore dans la théorie des fonctions polycaloriques.

Le problème de l'analyticité a été posé d'une manière concrète dans une communication récente au $\mathrm{III}^{\mathrm{e}}$ Congrès Unional des Mathématiciens Sovietiques [6].

Le cadre le plus convenable, au schéma proposé me paraît être une algèbre normée, par exemple une algèbre de Banach, possédant un élément unitaire. D'ailleurs, dans les deux premiers $\S$, les résultats sont valables pour une algèbre quelconque.

Soit, donc, 73 une algèbre commutative à élément unitaire $e$. Nous utiliserons au cours de ce travail des opérateurs linéaires $A, B, \ldots, L, \ldots$, c'est-à-dire additifs et homogènes sur le corps $K$ des nombres complexes.

Pour un opérateur linéaire quelconque $A$, nous poserons $A^{0}=e$, $A^{1}=A, A^{n}=A\left(A^{n-1}\right), n=1,2,3, \ldots$.

Avec cela, la signification d'une opération telle que $A^{m} B^{n} \ldots L^{p}$ est claire.

À tout opérateur linéaire $A$ nous attacherons l'opérateur bilinéaire $B$, défini par l'égalité

$$
B(x, y)=A(x y)-x A y-y A x
$$

où $x \epsilon 93, y \in$ 73. Manifestement, $B(x, y)=B(y, x)$

Il peut arriver que l'on ait $B(x, y)=\emptyset$, quels que soient les éléments $x, y$. Alors $A$ s'appelle un opérateur de dérivation, ou plus simplement une dérivée algébrique de l'élément auquel il s'applique [1]. Nous laisserons de côté ce cas, qui a été amplement étudié ${ }^{1}$ ) récemment.

Dans la suite nous considérerons un opérateur $A$ linéaire, pour lequel sont vérifiées certaines conditions.

* Les résultats de ce Mémoire ont été présentés au Congrès des Mathématiciens Autrichiens, Vienne, 17-22 septembre 1956.

1) On consultera, à ce propos, avec profit le Mémoire [1] de M. J. Mikusiński. Studia Mathematica XVI 